\title{
What has the pandemic
}

\section{taught us about our infection} control measures?

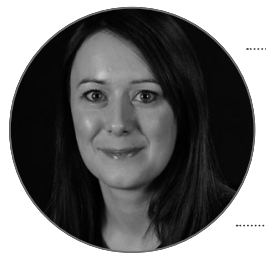

Rebecca Waters

Category Manager, Initial

Medical

he pandemic thus far has been a learning curve for many industries. Not only have

businesses and services had to

react to a fast-changing situation, but they have also had to evolve alongside it in order to survive.

Dentistry is no different, and though things are returning to a tentative normal, there are still a number of lessons that we have learned from the pandemic that can improve the industry moving forwards.
Perhaps the most significant of these lessons is that we must take a closer look at infection control measures in practice and what this means for the safety of patients and staff.

\section{Cleaning products need to be incredibly efficient}

When lockdown restrictions initially began to ease and dental practices were reopened, the first thing to do was to consider how to make these environments safe for patients and staff alike. Due to the novel nature of Coronavirus, research had to be carried out to see how it was spreading from person to person, and this meant that awareness of surface transmission rapidly increased as the topic came under the spotlight.

What was quickly clear was that, much like with other viruses and bacteria,
Coronavirus survives for different lengths of time on various surfaces. For example, research found that the virus lived for up to five days on ceramics and glass, but only lived for a number of hours on materials such as copper and aluminium. ${ }^{1}$ What this has taught us is that there is a need for a multi-pronged approach in practice when it comes to preventing disease transmission, and that cleaning products need to be suitable and effective on all surfaces.

Air quality is a concern that needs to be addressed

As studies into Coronavirus continued, one alarming find that emerged was that the virus could easily be spread in the air via aerosol. Though social distancing measures and masks had been put in place to help prevent 


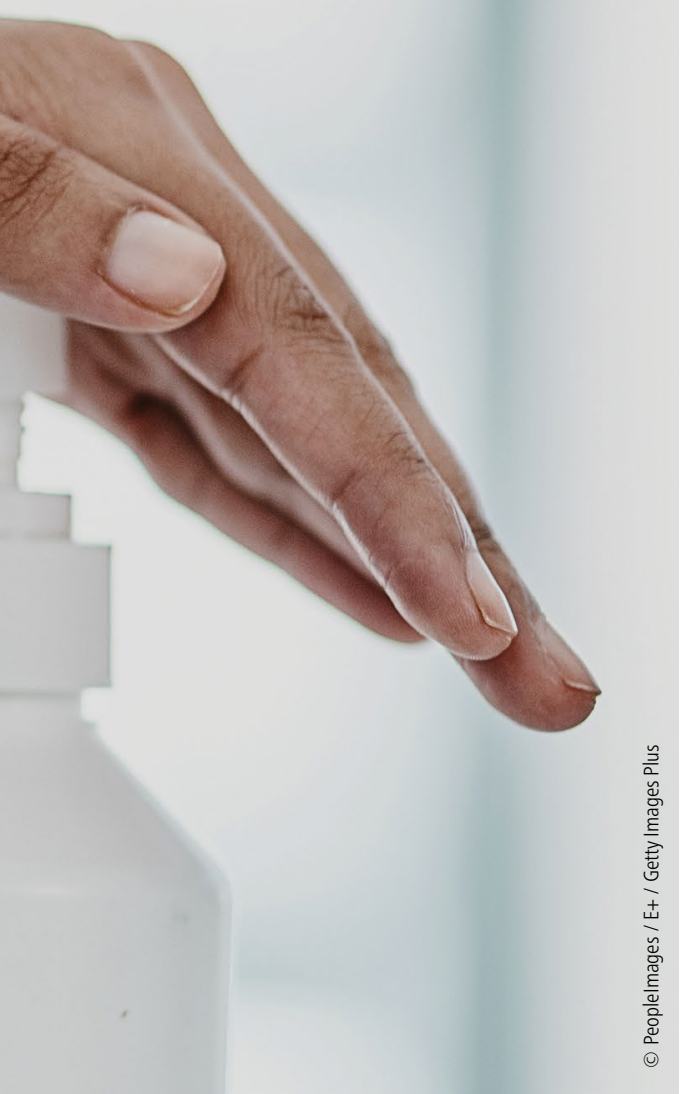

close contact airborne transmission, research later in the pandemic revealed that the virus could be spread further in the air, especially in places with poor ventilation or which saw a high footfall. ${ }^{2}$

As Coronavirus spreads very similarly to other diseases such as influenza, the understanding of airborne transmission is highly beneficial to guide how we perform infection control in the future. You may have already seen air purification technology for sale or disinfectants that release a cloud of solution that eradicates pathogens from the air - these are innovations that all practices should be looking towards, especially as they are very effective against all illnesses that could potentially be spread in this manner. By investing in an air purifier, you're helping to protect patients and staff against future illnesses, especially once/if face masks become no longer mandatory.

\section{Could higher levels of PPE become the norm?}

The use of PPE throughout the pandemic has increased enormously due to government guidance and individuals wanting to better protect themselves. However, while there are some perceived downsides to enhanced levels of PPE - such as the impact that this is having on the environment - there is also a good argument to be made for keeping these measures in place in the future.

\section{'As Coronavirus spreads very}

similarly to other diseases

such as influenza, the

understanding of airborne

transmission is highly

beneficial to guide how we

perform infection control in the future.'

PPE is a great first defence against all transmittable illnesses, and use of enhanced measures has had a significant impact on how well healthcare professionals have been shielded from the virus. ${ }^{3}$ It would, therefore, make sense for this to continue, especially if professionals are to defend against crosscontamination in practice.

Correct waste segregation and disposal have also become more vital than ever.

The pandemic revealed just how essential excellent waste management is, especially as a means to prevent cross contamination and limit disease transmission. Plus, the pandemic also introduced new waste streams that professionals had to learn about. Lateral flow tests, for example, are classed as chemical waste, and need to be disposed of accordingly.

\section{Patient and public education}

Perhaps one of the most useful consequences of the pandemic has been that people are now more alert to the need for infection control than ever before. This is positive news, as they are also more likely to follow measures that help prevent disease transmission, such as effective handwashing.

There is also the opportunity for professionals to speak to patients candidly about infection control and to encourage people to think about what this means for the future. People may naturally be more cautious after the Coronavirus outbreak, and this could mean that any future pandemics are handled much more simply as more people will be willing to comply with guidance.

\section{Preparing for the future}

Moving into the future, it makes sense to ensure that infection control measures in dental practices are as effective as possible. The introduction of enhanced PPE and air disinfectants are a step forward, and with the general public having an enhanced understanding of disease transmission and how to defend against it there's hope that we have all learned lessons that will benefit us in the future.

It's also likely that more and more practices will be working with companies such as Initial Medical, who not only supply a vast array of PPE items and a versatile range of infection control solutions that meet the current and future needs of professionals, but who are also experts in waste management and offer training to dental professionals to help ease the burden surrounding this allimportant subject.

With so many lessons learned from the pandemic, arguably one of the most significant takeaways has been that dentistry is a resilient industry that will always protect people and put them first. Even against the toughest challenges, dentistry has evolved and accommodated change to ensure that it remains an important asset in modern healthcare.

\section{References}

1. Web MD. How long Does Coronavirus Live On Surfaces? Available online at: www.webmd. com/lung/how-long-covid-19-lives-on-surfaces (Accessed April 2021)

2. World Health Organisation. Coronavirus Disease (COVID-19): How Is It Transmitted? Available online at: www.who.int/news-room/q-adetail/coronavirus-disease-covid-19-how-is-ittransmitted (Accessed April 2021).

3. Williams J. Enhanced PPE? Br Dent J 2020; 228 655-656

Rebecca has worked in the Healthcare sector for the past 17 years and was a Research Chemist with Bayer Cropscience prior to joining Rentokil Initial in 2003. She keeps up to date on all developments within the clinical waste management industry and is an active member of the CIWM, SMDSA and BDIA

https://doi.org/10.1038/s41404-021-0763-y 